\title{
Nonlinear Taylor vortices and their stability
}

\author{
By C. A. JONES \\ School of Mathematics, \\ University of Newcastle upon Tyne
}

(Received 13 February 1980 and in revised form 20 May 1980)

Axisymmetric numerical solutions of the Navier-Stokes equations for flow between rotating cylinders are obtained. The stability of these solutions to non-axisymmetric perturbations is considered and the results of these calculations are compared with recent experiments.

\section{Introduction}

In recent years, new experimental evidence has emerged concerning the classical problem of flow between rotating cylinders. Fenstermacher, Swinney \& Gollub (1979) and Donnelly et al. (1979) have studied flows for Reynolds numbers up to the transition to turbulence; this work has uncovered new modes of motion and extended the information on the well-established modes as described by, for example, Coles (1965).

The transition from azimuthal flow to axisymmetric toroidal vortices was first described experimentally and theoretically by Taylor (1923), and these axisymmetric vortices are called Taylor vortices: the linearized theory of this transition is well understood (e.g. Di Prima 1961; Roberts 1965; Krueger, Gross \& DiPrima 1966). The next transition occurs when the Taylor vortices become wavy; that is unstable to an out-of-phase non-axisymmetric disturbance. This transition has been discussed by Davey, DiPrima \& Stuart (1968). Their technique is based on expanding the solution in powers of the parameter $R e-R e_{\text {crit }}$, which is assumed small. This procedure was carried further by Eagles (1971), who also used it to find the torque on the cylinders (Eagles 1974). Numerical studies of this transition were performed by Meyer (1969).

Much of the recent experimental work has been aimed at understanding the transition to turbulence. This transition is not yet understood theoretically, but it has been discovered that sets of ordinary differential equations can give rise to Fourier time spectra closely resembling those obtained experimentally, and several sets of ordinary differential equations of varying complexity have been proposed (e.g. Rabinowitz 1978; Yahata 1979).

The approach used in this paper is to compute nonlinear, steady axisymmetric solutions of the Navier-Stokes equations which correspond to Taylor vortices. These solutions exist for all Reynolds numbers greater than critical, although they are not necessarily stable. We then consider small non-axisymmetric perturbations to these Taylor vortex solutions; i.e. we calculate the linear stability of these nonlinear axisymmetric solutions. This procedure is different from that of Davey et al. (1968) and Eagles (1971); these workers considered finite-amplitude perturbations, both axisymmetric and non-axisymmetric, to the state of purely azimuthal flow. While their method 
works well for Reynolds numbers just above critical, at larger Reynolds numbers it is no longer possible to use weakly nonlinear theory. The present approach is valid near the stability boundary of the onset of waviness; for some values of $\eta$, the radius ratio, this occurs at quite large Taylor number. Our method therefore permits some exploration of the high-Taylor-number regime without recourse to three-dimensional nonlinear computations. The physical justification for this approximation scheme is that the basic Taylor vortex motion persists to very high Reynolds numbers (Barcilon et al. 1979). The agreement between the predictions of the linearized theory presented here and the experimental results gives grounds for believing that many of the important observed phenomena in Taylor vortex flow can be treated in this way.

This technique requires the solution of partial differential equations in two spatial variables with prescribed boundary values. Many well-tried numerical methods are available for the solutions of such problems. Here we use the Galerkin method, which has been applied to similar convection problems by Clever \& Busse (1974) and Jones \& Moore (1978), and which here gives solutions accurate to better than $1 \%$ for Taylor numbers of up to about $50000\left(R e \simeq 5 R e_{\text {crit }}\right)$. In the Galerkin method the stability problem is solved by finding the eigenvalues of matrices related to the differential equations. Since routines are available for finding all the eigenvalues of matrices, the possibility of missing important eigensolutions is less than with iterative methods of solution. It is still unfortunately not practical to examine all types of perturbations, but we can use the labora tory experiments to guide us as to which types of disturbances are most likely to be unstable.

We have considered here only the case where the inner cylinder is rotating and the outer cylinder is at rest. The majority of the experiments relate to this case. We have also assumed that the cylinders have infinite length; some interesting effects are thereby omitted (Benjamin 1978). It was quickly realized that varying the gap ratio, $\eta=R_{\text {inner }} / R_{\text {outer }}$, has a profound effect on the character of the problem; even a gap ratio of 0.9 is substantially different from a narrow gap. Most attention was paid to two cases; $\eta=0.8756$, close to that of many of the experiments, and the 'narrow gap' approximation, $\eta \rightarrow 1$.

\section{Basic equations}

For the nonlinear equilibrium equations, the axisymmetric incompressible NavierStokes equations can be conveniently formulated in the cylindrical polar co-ordinate system $(r, \phi, z)$, where $z$ is parallel to the rotation axis. The velocity field is

$$
\mathbf{u}=\left(u_{r}, u_{\phi}, u_{z}\right)=\left(-\frac{1}{r} \frac{\partial \psi}{\partial z}, u_{\phi}, \frac{1}{r} \frac{\partial \psi}{\partial r}\right) .
$$

Before the onset of Taylor vortices, the velocity field is azimuthal,

$$
u_{\phi}=\frac{\Omega_{0} R_{1}^{2}}{d\left(R_{1}+R_{2}\right)}\left[\frac{R_{2}^{2}}{r}-r\right]=u_{\phi}^{0}(r),
$$

where $R_{1}$ and $R_{2}$ are the radii of the inner and outer cylinders, respectively, $d=R_{2}-R_{1}$ and $\Omega_{0}$ is the angular velocity of the inner cylinder. After the Taylor transition it is convenient to define

$$
v=u_{\phi}-u_{\phi}^{0}(r)
$$


so that $v$ is the departure of the azimuthal velocity field from the basic Couette flow state. The azimuthal vorticity equation is

where

$$
\frac{\partial(\psi, Z)}{\partial(r, z)}-\frac{2}{r}\left(u_{\phi}^{0}+v\right) \frac{\partial v}{\partial z}=\nu\left(r \frac{\partial^{2} Z}{\partial z^{2}}+\frac{\partial}{\partial r} \frac{1}{r} \frac{\partial}{\partial r}\left(r^{2} Z\right)\right)
$$

is the potential vorticity,

$$
Z=-\frac{1}{r} \frac{\partial}{\partial r} \frac{1}{r} \frac{\partial \psi}{\partial r}-\frac{1}{r^{2}} \frac{\partial^{2} \psi}{\partial z^{2}}
$$

$$
\frac{\partial(\psi, Z)}{\partial(r, z)} \equiv \frac{\partial \psi}{\partial r} \frac{\partial Z}{\partial z}-\frac{\partial \psi}{\partial z} \frac{\partial Z}{\partial r}
$$

is the Jacobian of $\psi$ and $Z$ (ef. Jones, Moore \& Weiss 1976) and $\nu$ is the kinematic viscosity. The azimuthal momentum equation is then

$$
\frac{\partial(\psi, v)}{\partial(r, z)}=\frac{v}{r} \frac{\partial \psi}{\partial z}+\frac{1}{r} \frac{\partial \psi}{\partial z} \frac{\partial}{\partial r}\left(r u_{\phi}^{0}\right)+v\left(\frac{\partial}{\partial r} r \frac{\partial v}{\partial r}+r \frac{\partial^{2} v}{\partial z^{2}}-\frac{v}{r}\right)
$$

The boundary conditions $u_{r}=v=u_{z}=0$ on $r=R_{1}$ and $R_{2}$ imply that

$$
\psi=\frac{\partial \psi}{\partial r}=v=0
$$

at these boundaries, so (2.4) and (2.5) constitute a sixth-order system in $r$ and $z$.

We now adopt the narrow-gap scalings (see, for example, Krueger et al. 1966) to get the equations into dimensionless form in such a way that all quantities remain finite in the limit $\eta \rightarrow 1$. We introduce $x, \zeta$ defined by $z=\zeta d$ and $r=R_{1}+x d$. The equations (2.4) and (2.5) become, in terms of the dimensionless variables,

where

$$
\begin{gathered}
\frac{\partial(\psi, Z)}{\partial(x, \zeta)}-\frac{T a \eta}{1-\eta}\left(\frac{1}{\hat{r}^{2}}-1\right) \frac{\partial v}{\partial \zeta}-\frac{T a(1+\eta)}{\hat{r}} v \frac{\partial v}{\partial \zeta}=\hat{r}\left[\frac{\partial^{2} Z}{\partial \zeta^{2}}+\frac{\partial}{\partial x} \frac{1}{\hat{r}} \frac{\partial}{\partial x}\left(\hat{\gamma}^{2} Z\right)\right], \\
\frac{\partial(\psi, v)}{\partial(x, \zeta)}+\frac{2 \eta}{1+\eta} \frac{\partial \psi}{\partial \zeta}-\frac{(1-\eta)}{\hat{r}} v \frac{\partial \psi}{\partial \zeta}=\frac{\partial}{\partial x} \hat{r} \frac{\partial v}{\partial x}+\hat{r} \frac{\partial^{2} v}{\partial \zeta^{2}}-\frac{(1-\eta)^{2}}{\hat{r}} v
\end{gathered}
$$

$$
Z=-\frac{1}{\hat{r}} \frac{\partial}{\partial x} \frac{1}{\hat{r}} \frac{\partial \psi}{\partial x}-\frac{1}{\hat{\gamma}^{2}} \frac{\partial^{2} \psi}{\partial \zeta^{2}}, \quad \hat{r}=\eta+x(1-\eta), \quad T a=\frac{2 \Omega^{2} d^{3} R_{1}^{2}}{\nu^{2}\left(R_{1}+R_{2}\right)}=\frac{2 \Omega^{2} d^{4} \eta^{2}}{\nu^{2}\left(1-\eta^{2}\right)} .
$$

This definition of $T a$ is not standard, as a variety of definitions of $T a$ have appeared in the literature. The Reynolds number $R e=\Omega_{0} R_{1} d / \nu=((1+\eta) T a / 2(1-\eta))^{\frac{1}{2}}$. The narrow-gap equations can now be simply obtained from (2.7) and (2.8) by letting $\eta \rightarrow 1$.

The torque on the inner and outer cylinders in these units is given by

$$
\begin{gathered}
G_{\text {Inner }}=G_{0}\left[\frac{2}{\eta(1+\eta)}-\left.\frac{\partial v}{\partial x}\right|_{x=0}\right] ; \quad G_{\text {outer }}=G_{0}\left[\frac{2}{\eta(1+\eta)}-\left.\frac{1}{\eta^{2}} \frac{\partial v}{d x}\right|_{x=1}\right] \\
G_{0}=\frac{2 \pi R_{1}^{3} h v \rho \Omega_{0}}{d},
\end{gathered}
$$

where $h$ is the length of the cylinders and $\rho$ the fluid density. In a steady state

$$
G_{\text {outer }}=G_{\text {inner }}=G \text {. }
$$


Small disturbances to the equilibrium flow given by (2.7) and (2.8) satisfy

$$
\frac{\partial \mathbf{u}^{\prime}}{\partial t}+\left(\mathbf{u}^{0} \cdot \nabla\right) \mathbf{u}^{\prime}+\left(\mathbf{u}^{\prime} \cdot \nabla\right) \mathbf{u}^{0}=-\nabla p^{\prime}+\nu \nabla^{2} \mathbf{u}^{\prime}
$$

and

$$
\nabla \cdot \mathbf{u}^{\prime}=0 \text {. }
$$

For non-axisymmetric disturbances we can eliminate $u_{\phi}^{\prime}$ via (2.11) and obtain equations with $u_{r}^{\prime}$ and $u_{z}^{\prime}$ as dependent variables. We use the narrow-gap scalings, so that $\phi$ is scaled by writing $\phi=\left(\nu / \Omega_{0} d^{2}\right) \phi$. If disturbances are proportional to $e^{i m \phi}$, we introduce $k$ such that $k \hat{\phi}=m \phi$, which means $k=m\left(T a\left(1-\eta^{2}\right) / 2 \eta^{2}\right)^{\frac{1}{2}}$. For a finite gap, $m$ must be an integer; as $\eta \rightarrow 1$ in the narrow-gap approximation, $k$ must remain finite. The interval between the successive discrete values of $k$ therefore becomes small.

We eliminate the pressure from (2.10) by using the $r$ and $\phi$ components of the vorticity equation to give us the required equations. The equations are too complicated to give details, although they are of the form

$$
\left.\begin{array}{l}
(\sigma-i \omega) L_{1}\left(u_{r}^{\prime}, u_{z}^{\prime}\right)=M_{1}\left(u_{r}^{\prime}, u_{z}^{\prime}, \psi, v\right), \\
(\sigma-i \omega) L_{2}\left(u_{r}^{\prime}, u_{z}^{\prime}\right)=M_{2}\left(u_{r}^{\prime}, u_{z}^{\prime}, \psi, v\right),
\end{array}\right\}
$$

where disturbances are proportional to $e^{(\sigma-i \omega) t}(\sigma, \omega$ real $)$ and $L_{1}, L_{2}, M_{1}$ and $M_{2}$ are differential operators, linear in $u_{r}^{\prime}$ and $u_{z}^{\prime}$. The boundary conditions are

$$
u_{z}^{\prime}=u_{r}^{\prime}=\frac{\partial u_{r}^{\prime}}{\partial x}=0
$$

so that determining the stability of our axisymmetric equilibrium is a sixth-order eigenvalue problem for $\sigma-i \omega$ in the two independent variables $x$ and $\zeta$.

\section{Steady axisymmetric solutions}

To solve equations (2.7) and (2.8) we expand $\psi$ and $v$ as a Fourier series in $\zeta$. We look for solutions such that adjacent Taylor vortices are separated by planes, so

$$
\psi=\sum_{n=1}^{\infty} \psi_{n}(x) \sin n \alpha \zeta, \quad v=\sum_{n=0}^{\infty} v_{n}(x) \cos n \alpha \zeta
$$

We now make the further expansions

$$
\psi_{n}(x)=\sum_{m=0}^{\infty} \psi_{m n} T_{m}^{*}(x), \quad v_{n}(x)=\sum_{m=0}^{\infty} v_{m n} T_{m}^{*}(x)
$$

where the $T_{m}^{*}(x)$ are the reduced Tchebycheff polynomials (see, for example, Fox \& Parker 1968). In order to obtain a numerical solution, the infinite sums (3.1) and (3.2) are truncated after $n=N$ and $m=M$ terms, respectively. When these finite sums are substituted into the boundary conditions (2.6), $6 N+2$ algebraic equations result from equating the coefficients of $\sin n \alpha \zeta$ and $\cos n \alpha \zeta$ to zero. Since we have $(M+1)(2 N+1)$ unknown coefficients from the $\psi_{m n}$ and $v_{m n}$ we need a further $(M-3) N+(M-1)(N+1)$ algebraic equations to be derived from equations (2.7) and (2.8). There are several different ways of obtaining these equations. In the Lanczos $\tau$ method (Fox \& Parker 1968) we substitute the expansions (3.1) and (3.2) into the partial differential equations and equate the coefficients of $T_{m}^{*}(x) \sin n \alpha \zeta$ for $m=0, M-4$ and $n=1, N$ to zero in 
(2.7) and the coefficients of $T_{m}^{*}(x) \cos n \alpha \zeta$ for $m=0, M-2$ and $n=0, N$ to zero in (2.8). In this way we obtain $(M-3) N$ equations from (2.7) and $(M-1)(N+1)$ equations from (2.8), giving us the required total. The resulting algebraic equations are then solved using Newton-Raphson iteration. The nonlinear terms consist of products of sums which have to be written as a sum of the form

$$
\Sigma a_{m n} T_{m}^{*}(x)\left\{\begin{array}{l}
\sin n \alpha \zeta \\
\cos n \alpha \zeta
\end{array}\right\}
$$

In the finite-gap case this procedure becomes rather cumbersome as the coefficients involve rather complicated functions of $x$. We therefore adopt a collocation procedure (see, for example, Wright 1964) in the $x$ direction. In this method, we require that the coefficients of $\sin n \alpha \zeta$ in equation (2.7) are equated to zero at the $M-3$ points given by the zeros of $T_{M-3}^{*}(x)$; similarly, the coefficients of $\cos n \alpha \zeta$ in equation (2.8) are equated to zero at the $M-1$ points given by the zeros of $T_{M-1}^{*}(x)$. Collocation is simpler to perform, but for given $M$ and $N$ is not quite as accurate for this problem. Comparison of the two methods in the narrow-gap case indicated that both methods give similar accuracy for the same computer time. The collocation method is much easier to program.

Even if $\alpha$ is prescribed, there is in general no uniqueness of solution. When $T a$ is just above critical, only one solution was found closely corresponding to the form of the first eigenfunction of the linear problem. At higher $T a$, linear solutions corresponding to multiple rolls in the radial and axial directions become unstable, and nonlinear solutions corresponding to these solutions exist. Care was taken to ensure that the results presented here all relate to the stability of the single-roll solution, which corresponds to the solution of the linearized axisymmetric problem with the smallest critical Taylor number. We choose $\alpha=3.13$ which is close to that value which gives minimum critical Taylor number for $0.75 \leqslant \eta<1$, except in the comparison with Rogers \& Beard (1969) where $\eta=0.5$ and we use $\alpha=3 \cdot 1631$.

The value of $\alpha$ is given experimentally by the number of cells that fit into the Couette apparatus. This number is not uniquely determined (e.g. Coles 1965); for example, Gorman \& Swinney (1980) found stable states with $16,17,18,19$ and 20 cells in an apparatus with aspect ratio 20, corresponding to values of $\alpha$ ranging from $2 \cdot 5$ to $\pi$. The results presented here are not generally very sensitive to changes in $\alpha$ of this order. We note that to maximize the torque would require values of $\alpha$ greater than 3.13, so choosing $\alpha$ to maximize torque would be inappropriate (DiPrima \& Eagles 1977).

The numerical results for the torque were in agreement with those of Meyer (1969), Rogers \& Beard (1969) and, in the range when finite-amplitude expansions hold, with Davey (1962). In table 1 we compare various calculations of the non-dimensional torque for the case $\eta=0.5$. We show how our calculations compare with the numerical calculations of Rogers \& Beard (1969), who used finite-differences with 40 mesh points in the radial direction and Fourier expansion with truncation at $N=6$ in the axial direction. We also give the values found by Davey's (1962) amplitude expansion and the experiments of Donnelly \& Simon (1960). To give some idea of how the accuracy depends on the truncation parameters $M$ and $N$ we show the present results with $M \times N=13 \times 6,13 \times 7$ and $14 \times 6$. In table 1 all calculations have $\eta=0 \cdot 5, \alpha=3 \cdot 1631$; $T a_{\text {crit }}$ for these values is $3099 \cdot 6$.

Figure 1 shows the torque as a function of Reynolds number for $\alpha=3 \cdot 13$ in the 


\begin{tabular}{|c|c|c|c|c|c|c|}
\hline \multirow[b]{2}{*}{$T a$} & \multicolumn{3}{|c|}{$\mathbf{M} \times \mathbf{N}$} & \multirow{2}{*}{$\begin{array}{l}\text { Rogers } \\
\text { \& Beard }\end{array}$} & \multirow[b]{2}{*}{ Davey } & \multirow{2}{*}{$\begin{array}{l}\text { Donnelly } \\
\text { \& Simon }\end{array}$} \\
\hline & $13 \times 6$ & $13 \times 7$ & $14 \times 6$ & & & \\
\hline 3500 & $2 \cdot 813013$ & $2 \cdot 813013$ & $2 \cdot 813015$ & $2 \cdot 81$ & $2 \cdot 81$ & $2 \cdot 80$ \\
\hline 7500 & 3.587817 & 3.587816 & 3.587786 & 3.59 & $3 \cdot 40$ & $\mathbf{3 . 5 0}$ \\
\hline 15000 & $4 \cdot 1861$ & $4 \cdot 1870$ & $4 \cdot 1858$ & $4 \cdot 16$ & $3 \cdot 65$ & 4.09 \\
\hline 30000 & 4.8771 & $4 \cdot 8053$ & 4.7777 & - & - & $4 \cdot 74$ \\
\hline
\end{tabular}

TABLE 1. Dimensionless torque $G / G_{0}$.

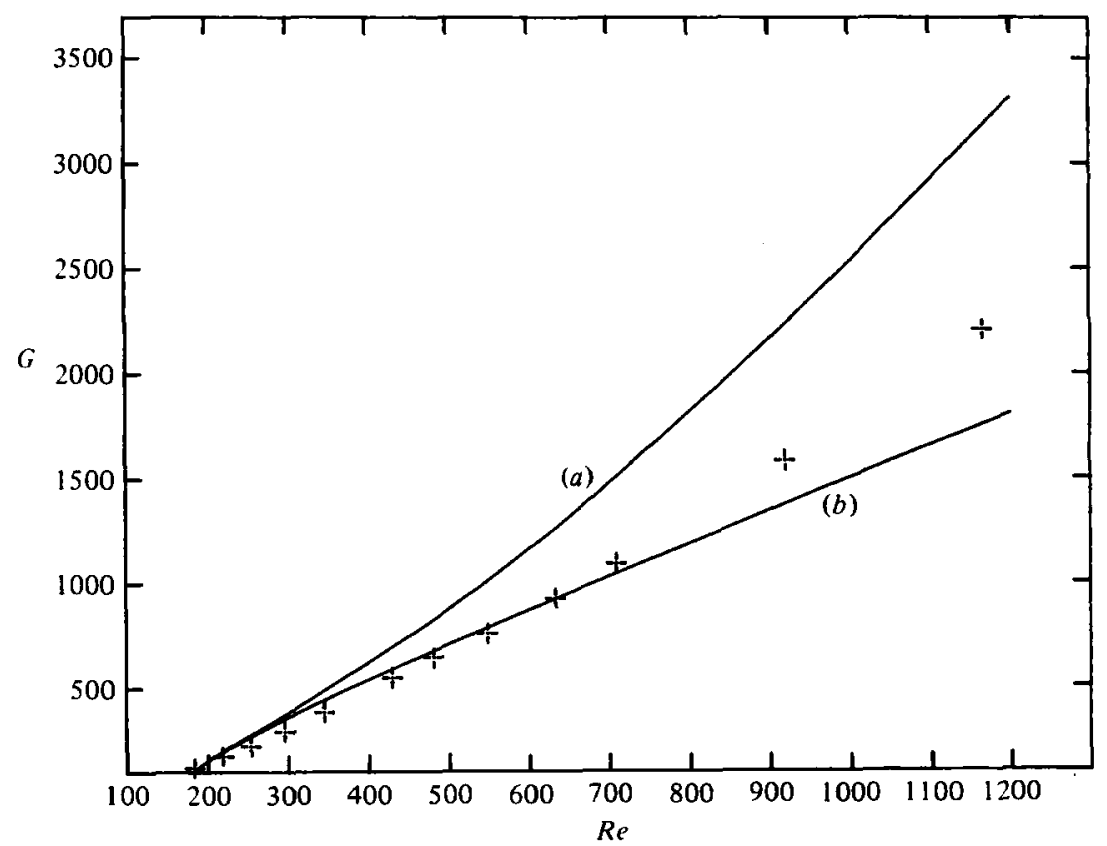

Figure 1. The torque, $G$, is plotted against Reynolds number, Re. Curve (a) uses the nonlinear axisymmetric solution with $\eta=0.95$ and axial wavenumber $\alpha=3.13$. (b) is the curve obtained with the amplitude expansion of Davey (1962). +++ , Experimental results of Donnelly \& Simon (1960).

$\eta=0.95$ case, using the full finite-gap equations. Also shown is the equivalent curve from Davey (1962) and the experimental results of Donnelly \& Simon (1960). As noted by Meyer (1969), the predicted torque is considerably larger than the observed torque. Eagles (1974) showed that at fixed $\alpha$ the presence of waviness in the cells reduced the torque and for $m=4$ obtained good agreement with observation near $R e=R e_{\text {crit }} ;$ Meyer (1969) showed that for purely axisymmetric cells decreasing $\alpha$ also reduced the torque. In order to obtain agreement with experimental values purely by decreasing $\alpha$, a value of $\alpha=1.8$ in the axisymmetric solution is required. The numerical experiments are in reasonable agreement with Batchelor's (1960) asymptotic torque law; a $\log$ - $\log$ plot of the numerical experiments gives $G \propto R e^{1.52}$ at high Reynolds numbers compared with Batchelor's law of $G \propto R e^{1 \cdot 5}$. 
(a)

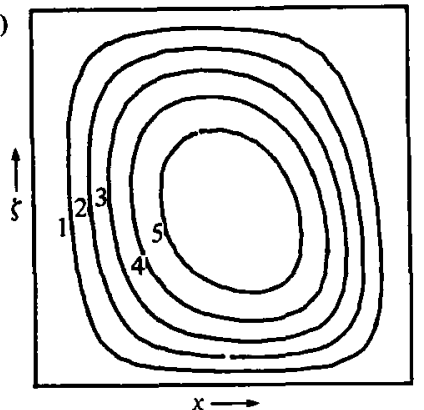

(b)

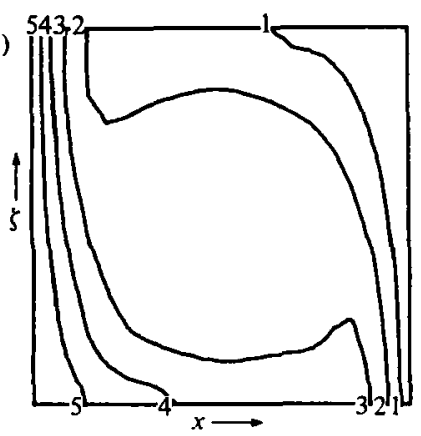

(c)

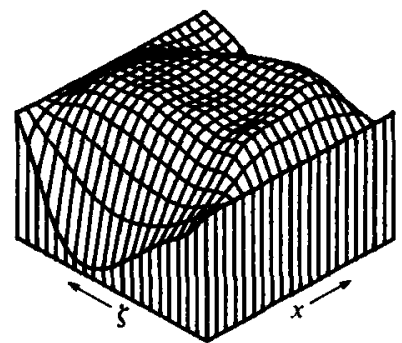

(d)

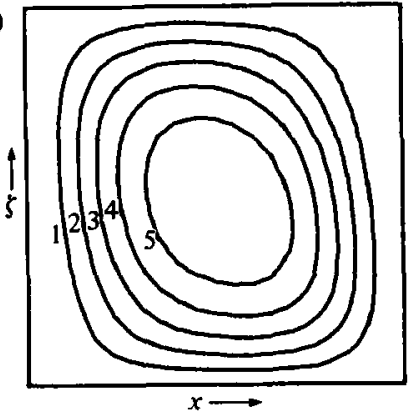

(e)

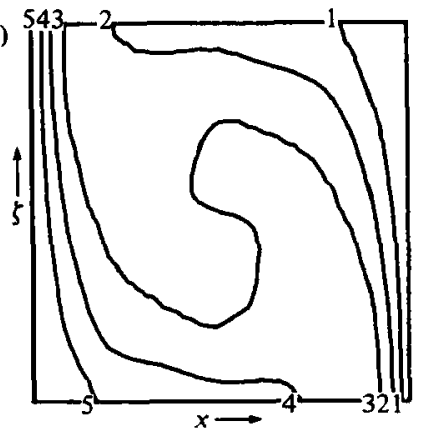

$(f)$

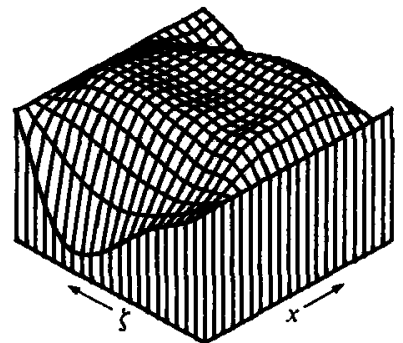

Fiaure 2. (a) Streamlines, (b) contours of equal azimuthal velocity, (c) potential vorticity distribution for the narrow-gap case with $T a=50000, \alpha=3.13$. In the contour plots, the contours are equally spaced. $(d),(e)$ and $(f)$ are the analogous pictures in the case $\eta=0.8756$.

In figure 2 the streamlines, potential vorticity and azimuthal velocity contours are shown. It is interesting to compare with the analogous pictures for Bénard convection (e.g. Moore \& Weiss 1973). Both cases shown are for $T a=50000, \alpha=3 \cdot 13$ : cases $(a)$ to $(c)$ are for narrow gaps and $(d)$ to $(f) \eta=\mathbf{0 . 8 7 5 6}$. The $\eta=0 \cdot 8756$ case displays more asymmetry than the narrow-gap case; thus the speed of the inviscid core is 0.5 times the speed of the inner cylinder in the narrow-gap case, but is considerably less in the case $\eta=\mathbf{0 . 8 7 5 6}$. Also of note is the flat distribution of potential vorticity in the core region (Batchelor 1956). 


\section{Small perturbations to the Taylor vortices}

We now consider disturbances to the axisymmetric solutions proportional to $e^{i m \phi}$, or, in the narrow-gap case, $\propto e^{i k \hat{\phi}}$. We write

$$
\left.\begin{array}{l}
u_{\mathfrak{z}}^{\prime}=\left[\sum_{n=1}^{\infty} W_{n}^{i}(x) \sin n \alpha \zeta+\sum_{n=0}^{\infty} W_{n}^{0}(x) \cos n \alpha \zeta\right] e^{i m \phi}, \\
u_{r}^{\prime}=\left[\sum_{n=0}^{\infty} V_{n}^{i}(x) \cos n \alpha \zeta+\sum_{n=1}^{\infty} V_{n}^{0}(x) \sin n \alpha \zeta\right] e^{i m \phi},
\end{array}\right\}
$$

so we are considering only disturbances which have the same axial period as the Taylor cells. On substituting these into the equations (2.12) and (2.13), the stability problem separates into two parts; the in-phase modes $\mathbf{u}^{i}$ and the out-of-phase modes $\mathbf{u}^{0}$. Since there is no interaction between these modes, we solve the two cases separately.

In both cases, $W_{n}$ and $V_{n}$ are expanded in a Tchebycheff series, and the resulting expansions are put into equations (2.12). As with the axisymmetric equations we used the Lanczos $\tau$ method for the narrow-gap cases and collocation for finite gaps. In either case, the system is truncated at $n=N$ and $m=M$, and the number of unknowns is reduced by applying the boundary conditions (2.13). The coefficients $W_{m n}$ and $V_{m n}$ are put together to create a single vector $a_{i}$, and the linear eigenvalue problem then has the form

$$
(\sigma-i \omega) L \mathbf{a}=M \mathbf{a}
$$

where $L, M$ are complex matrices. These matrix equations were solved using routines in the NAG (Numerical Algorithm Group) library, reference numbers F04ADF and F02AJF. When required, the eigenvectors $a_{j}$ can be found using routine F02AKF and the velocity fields corresponding to the perturbation can be obtained. Values of $M$ up to 14 and $N$ up to 8 were found to be practical: this enables the eigenvalues to be found for values of $T a$ up to 50000 (Re $\left.\simeq 5 R e_{\text {crit }}\right)$. The upper limit to $M$ and $N$ is determined principally by the storage requirement. When the basic flow was purely aximuthal, the program gave results which agreed with those of Roberts (1965).

The out-of-phase modes are the most important, as found by Davey et al. (1968). These perturbations turn the axisymmetric vortices into wavy vortices. In figure 3 , the stability boundary for the onset of the wavy mode is shown. Also plotted is the corresponding curve for the onset of Taylor vortices from Roberts (1965). As is clear from figure 3 , the usual wavy mode of instability is not found when $\eta<0.75$ and $T a<12500$. At $T a=24000$ the stability boundary is at $\eta=0.77$; at $T a$ much larger than this the program is no longer sufficiently accurate to determine $\eta$ to better than $1 \%$. For $\eta>0.8$ the $m=1$ mode is the first to become unstable, but for $T a>7000$ and $\eta<0.77$ the $m=3$ mode determines stability. Also shown are the experimental results of Zarti \& Mobbs (1979) for the appearance of 'strong vortex waves'. They also have a point at $T a=24 T a_{\text {crit }}, \eta=0.725$ which is at too high a Taylor number for reliable calculation by this method, but does indicate that the rapid increase in $T a$ for the onset of waviness as $\eta$ is reduced from 0.8 to 0.7 predicted by these calculations is correct. It would be of interest to obtain more experimental information for cylinders with $\eta$ between 0.7 and 0.8 to compare with figure $3:$ such experiments must, of course, have large aspect ratio for meaningful comparison. 


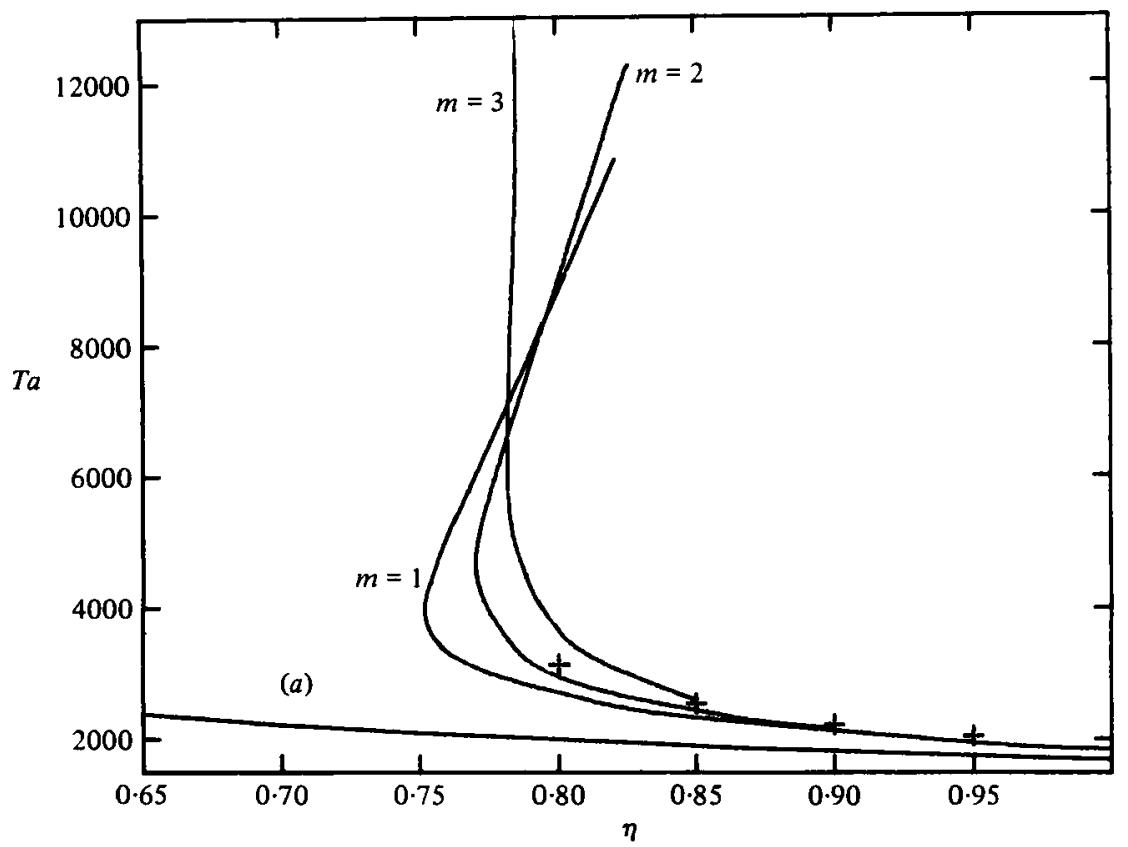

FIGURE 3. Curve $(a)$ is the stability boundary for the onset of axisymmetric Taylor vortices (Roberts 1965). The curves $m=1, m=2$ and $m=3$ are curves of neutral stability for the nonaxisymmetric modes; in the region above and to the right of the envelope of these curves the vortices are wavy. The axial wavenumber $\alpha=3 \cdot 13$.

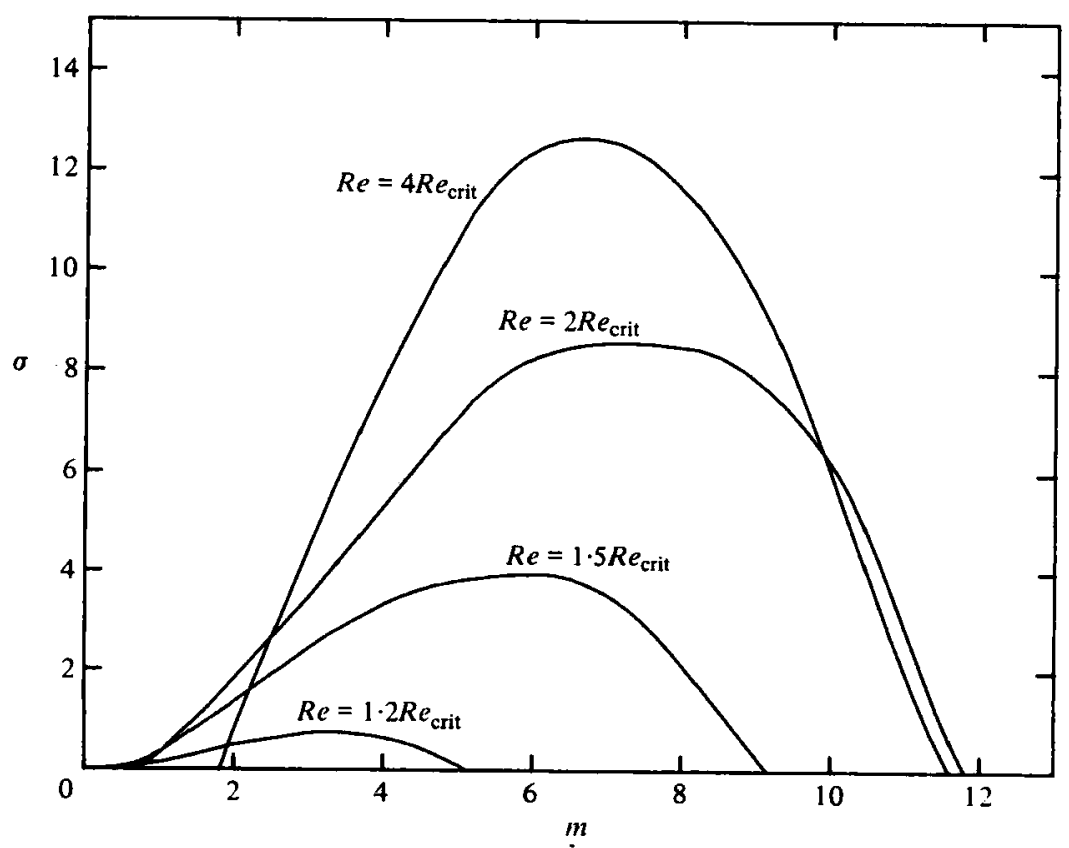

Figure 4. The growth rate, $\sigma$, is shown as a function of $m$, the azimuthal wavenumber. Curves for $R e=1.2 R e_{\text {crtt }}, 1.5 R e_{\text {crlt }}, 2 R e_{\text {crlt }}$ and $4 R e_{\text {crit }}$ are shown. $R e_{\text {erit }}=118.4$. Axial wavenumber $\alpha=3.13$ and $\eta=0.8756$ in all cases. $\sigma$ is measured in units of $y / d^{2}$. 


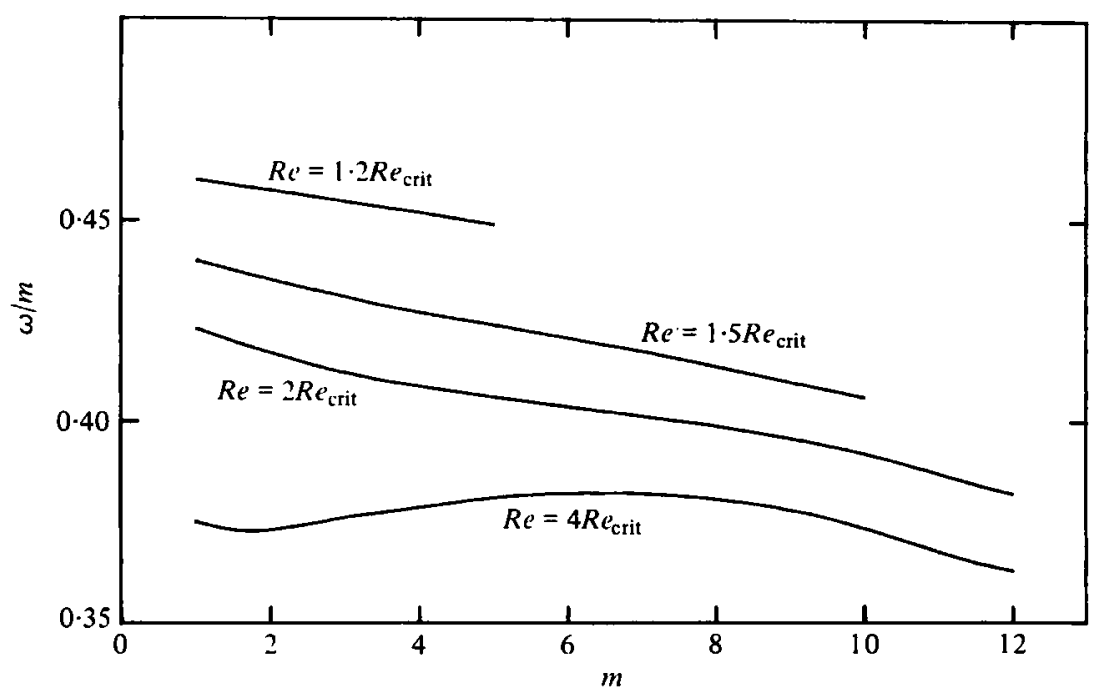

Fraure 5. The phase speed, $\omega / m$, is shown as a function of $m$, azimuthal wavenumber. Curves for $R e=1 \cdot 2 R e_{\text {crit }}, 1.5 R e_{\text {crit }}, 2 R e_{\text {crlt }}$ and $4 R e_{\text {crit }}$ are shown. Axial wavenumber, $\alpha=3.13$ and $\eta=0.8756$ in all cases. $\omega / m$ is measured in units of $\Omega_{0}$.

Although the longest waves are the first to become unstable for $\eta>0 \cdot 8$, as $T a$ is increased values of $m$ greater than 1 become the fastest-growing mode, and the number of unstable $m$ 's increases. In figure 4 we show the growth rate as a function of $m$ at various $T a$, for $\eta=0.8756$. The range of unstable modes $(m \lesssim 12)$ is in agreement with the range of modes observed experimentally (Donnelly 1979, private communication). As observed by Coles, at given $R e$ several states with differing $m$ can be found experimentally, so observations cannot pick out a 'preferred' mode; however, the positions of the maximum growth rate in figure 4 do correspond with commonly observed states (Donnelly et al. 1979; Fenstermacher et al. 1979).

In the narrow-gap case, the growth rates are considerably larger than for finite gaps. The $k$-value for maximum growth rate increases rapidly as $T a$ increases; indeed, the position of maximum growth rate tends to infinity at values of $T a$ below $2 T a_{\text {crit }}$. The reason for this behaviour appears to be that the $\phi$ derivatives in the viscous stress term are neglected in the narrow-gap approximation.

As $k \rightarrow 0$ in the narrow-gap approximation, $\sigma$, and $\omega \rightarrow 0$ for the wavy mode; this $k=0$ mode corresponds to a neutral out-of-phase axisymmetric mode. It corresponds to a shift of the whole Taylor vortex pattern by a small amount in the axial direction. For values of $\eta$ close to 1 , the $m=1$ mode can be described approximately as a shift of the vortex pattern up on one side of the cylinder and down on the other. If the cylinders have end-walls, this neutral axisymmetric solution is no longer possible, so it is perhaps not surprising that the wavy mode is strongly damped if the aspect ratio $h / d$ is not large (Cole 1976).

The phase speeds, $\omega / m$, of waves of differing $m$ are approximately constant. In figure 5 we therefore plot $\omega / m$, measured in units of $\Omega_{0}$, as a function of $m$, for various Reynolds numbers. The frequencies are in good agreement with the experiments of Coles, with $\eta=0.874$, and Donnelly with $\eta=0.8756$; at $R e=4 R e_{\text {crit }}$ we find a phase speed of $0.37 \Omega_{0}$ compared to the observed value of $0.35 \Omega_{0}$. Since we are ignoring the 
nonlinearities associated with the non-axisymmetric components of the motion, this agreement is somewhat surprising; a possible explanation is that it is the azimuthal speed of the inviscid core which principally determines the phase speed. The perturbations are approximately stationary in the frame of reference of this core.

In the narrow-gap case, this core moves with speed close to $\frac{1}{2} \Omega_{0}$, and this is reflected in the fact that the phase speeds are always near $\frac{1}{2} \Omega_{0}$ if the gap is sufficiently narrow. This has been confirmed experimentally by Donnelly (1979, private communication).

The in-phase modes were examined for the case $\eta=0.8756$ up to $R e=5 R e_{\text {crit }}$. No unstable in-phase modes were found, and no mode appeared to be moving towards instability.

Up to $R e=5 R e_{\text {crit }}$ in the case $\eta=\mathbf{0 . 8 7 5 6}$ only one unstable out-of-phase mode was found for given $m$, corresponding to the usual wavy mode. However, as $R e$ is increased in the neighbourhood of $5 R e_{\text {crit }}$ there is a second mode which is moving towards instability. The spatial structure of the mode is rather similar to that of the wavy mode, as is the location of fastest growth rate as a function of $m$. It is estimated that this mode will become unstable in the vicinity of $R e \simeq 10 R e_{\text {crit }}$; this mode may be connected with the $f_{2}$ mode (Gorman \& Swinney 1979). New numerical techniques will be needed to confirm this.

\section{Conclusion}

The method of approximating three-dimensional wavy vortices by perturbing about axisymmetric Taylor vortices has the merit of requiring only the solution of twodimensional partial differential equations. These equations can be solved quite accurately by numerical methods for a wide range of Taylor numbers. The results obtained here using this method show agreement in some detail with experimental results. In particular, the location of the stability boundary (figure 3) agrees with the results of Zarti \& Mobbs, although more detailed comparison would be desirable. The phase speeds and the location of maximum growth rate as a function of $m$ are also in satisfactory agreement with observation; it might be hoped that this agreement could be further improved if it proves possible to develop a weakly nonlinear theory. Also of note is the result that the phase speed is close to one half of the inner cylinder speed for very narrow gaps, in agreement with the recent observations of Donnelly (1979, private communication).

The most important result in this work seems to be the nature of the stability boundary between axisymmetric Taylor vortex flow and wavy vortex flow shown in figure 3 ; in particular the rapid rise of the boundary as $\eta$ moves down from 0.8 to 0.7 . This raises the possibility of finding amplitude equations to describe the development of the linear modes; such a procedure requires a 'small' parameter, so such equations would be valid near the stability boundary of figure 3 . This region covers an area at high Taylor number where many interesting phenomena are experimentally observed. This region of the $T a, \eta$ plane therefore represents an area where theory and experiment might come together.

I am indebted to Professor R. J. Donnelly for access to experimental results, some unpublished, and for many helpful comments. I am grateful for useful conversation with Professor J. T. Stuart. I also acknowledge the co-operation of the Newcastle 
University Computing service for the use of the IBM 370/168 on which the computations were performed.

\section{REFERENCES}

Barcilon, A., Brindeey, J., Lessen, M. \& Mobbs, F. R. 1979 Marginal instability in TaylorCouette flows at a very high Taylor number. J. Fluid Mech. 94, 453-463.

Batchelor, G. K. 1956 On steady laminar flow with closed streamlines. J. Fluid Mech. 1, 177190.

Batchelor, G. K. 1960 Appendix to An empirical torque relation for supercritical flow between rotating cylinders (by R. J. Donnelly \& N. J. Simon). J. Fluid Mech. 7, 416-418.

Benjamin, T. B. 1977 Bifurcation phenomena in steady viscous flows of a viscous fuid. I. Theory; II. Experiments. Proc. Roy. Soc. A 359, 1-26; 27-43.

Clever, R. M. \& Busse, F. H. 1974 Transition to time-dependent convection. J. Flesid Mech. $65,625-645$.

CoLE, J. A. 1976 Taylor vortex instability and annulus-length effects. J. Fluid Mech. 75, 1-15.

Coles, D. 1965 Transition in circular Couette flow. J. Fluid Mech. 21, 385-425.

DAvey, A. 1962 The growth of Taylor vortices in flow between rotating cylinders. J. Fluid Mech. 14, 336-368.

Davey, A., DiPrima, R. C. \& STUART, J. T. 1968 On the instability of Taylor vortices. J. Fluid Mech. 31, 17-52.

DiPrima, R. C. 1961 Stability of nonrotationally symmetric disturbances for viscous flow between rotating cylinders. Phys. Fluids 4, 751-755.

DiPrima, R. C. \& Eagles, P. M. 1977 Amplification rates and torque for Taylor-vortex flows between rotating cylinders. Phys. Fluids 20, 171-175.

Donnelty, R. J. \& Srmon, N. J. 1960 An empirical torque relation for supercritical flow between rotating cylinders. $J$. Fluid Mech. 7, 401-418.

Donnelity, R. J. \& Schwarz, K. W. 1965 Experiments on the stability of viscous flow between rotating cylinders. Proc. Roy. Soc. A 283, 531-546.

Donnelly, R. J., Park, K., Shaw, R. \& Walden, R. W. 1979 Early non-periodic transitions in Couette flow. (In Press.)

EAGLEs, P. M. 1971 On stability of Taylor vortices by fifth-order amplitude expansions. J. Fluid Mech. 49, 529-550.

Eagles, P. M. 1974 On the torque of wavy vortices. J. Fluid Mech. 62, 1-9.

Fenstermacher, P. R., Swinney, H. L. \& GollUb, J. P. 1979 The transition to chaotic Taylor vortex flow. J. Fluid Mech. 94, 103-127.

Fox, L. \& PArker, I. B. 1968 Chebyshev Polynomials in Numerical Analysis. Oxford University Press.

Gonman, M. \& Swinney, H. L. 1979 Visual observation of the second characteristic mode in a quasi-periodic flow. Phys. Rev. Lett. 43.

Jones, C. A., Moore, D. R. \& WeIss, N. O. 1976 Axisymmetric convection in a cylinder. J. Fluid Mech. 73, 353-388.

Jonks, C. A. \& Moore, D. R. 1979 Stability of axisymmetric convection. Geophys. Astrophys. Fluid Dyn. 11, 245-270.

Krueger, E. R., Gross, A. \& DiPrima, R. C. 1966 On the relative importance of Taylor-vortex and non-axisymmetric modes in flow between rotating cylinders. J. Fluid Mech. 24, 521-538.

MEYer, K. A. 1969 Thre日-dimensional study of flow between concentric rotating cylinders. In High Speed Computing in Fluid Dynamics. Phys. Fluids Suppl. II12, 165-170.

MooRe, D. R. \& WeIss, N. O. 1973 Two-dimensional Rayleigh-Bénard convection. J. Fluid Mech. 58, 289-312.

Rabinowitz, M. I. 1978 Stochastic self-oscillations and turbulence. Sov. Phys. Uspecki 21 (5), $443-469$.

Roberts, P. H. 1965 Appendix to Experiments on the stability of viscous flow between rotating cylinders. VI. Finite amplitude experiments. Proc. Roy. Soc. A 283, 531-556. 
Rogers, E. H. \& BEARd, D. W. 1969 A numerical study of wide gap Taylor vortices. J. Comp. Phys. 4, 1-18.

TAYlor, G. I. 1923 Stability of a viscous liquid contained between two rotating cylinders. Phil. Trans. Roy. Soc. A 223, 289-343.

WRIGHT, K. 1964 Chebyshev collocation methods for ordinary differential equations. Computer $J .6,358-365$.

Yahata, H. 1978 Temporal development of the Taylor vortices in a rotating fluid. Prog. Theor. Phys. Suppl. 64, 165-185.

ZARTI, A. S. \& MoBBs, F. R. 1979 Wavy Taylor vortex flow between eccentric rotating cylinders. Energy conservation through fluid film lubrication technology: Frontiers in Research and Design. A.S.M.E. 